\title{
Vaisseaux sanguins et tumeurs ou l'art du dialogue
}

$>$ Le réseau vasculaire joue un rôle essentiel dans le développement des cancers. Au cours de la dernière décennie, la découverte de cellules tumorales à caractère souche logées à proximité des vaisseaux sanguins a permis de mettre en lumière l'existence d'interactions fonctionnelles entre l'endothélium et les cellules tumorales. Dans cette revue, nous présentons les mécanismes d'angiogenèse, ainsi que les éléments de langage dans le dialogue établi entre la tumeur et son microenvironnement. Nous y détaillons, d'une part, les effets de la masse tumorale et son implication dans la modulation de la plasticité endothéliale et, d'autre part, la contribution de la niche vasculaire à la tumorigenèse. <

La progression et l'invasion tumorales sont des processus complexes au cours desquels les cellules acquièrent des propriétés aberrantes de prolifération, survie et migration, et la capacité à former un réseau sanguin dédié. Les vaisseaux tumoraux apportent, notamment, nutriments et oxygène aux cellules cancéreuses, et contribuent à la formation d'un microenvironnement spécifique dans lequel les cellules endothéliales de la paroi vasculaire et les cellules de la masse tumorale peuvent interagir. Des travaux récents ont permis de mettre en évidence l'existence d'interactions fonctionnelles bidirectionnelles entre l'endothélium et les cellules tumorales. Dans cette revue, nous nous intéressons à ces échanges et à leur contribution à la tumorigenèse.

\section{Morphogenèse vasculaire et développement tumoral}

\section{Développement vasculaire normal}

L'arbre vasculaire se forme selon deux processus différents: la vasculogenèse et l'angiogenèse. La vasculogenèse consiste en la différenciation des

Cet article fait partie du numéro thématique publié par médecine/sciences en avril 2014 et intitulé « Microenvironnement tumoral ».
Sandy Azzi, Julie Gavard

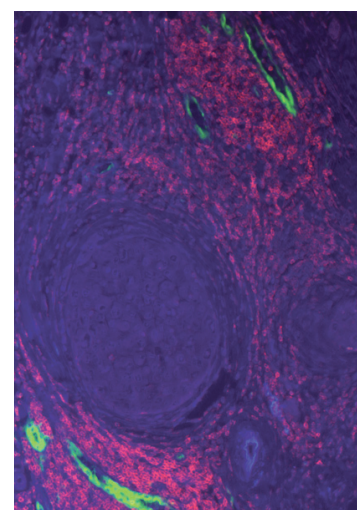

CNRS, UMR 8104, 22 , rue Mechain, Paris 75014, France ; Inserm, U1016, 22, rue Mechain, Paris 75014 , France ; université Paris Descartes, Sorbonne Paris Cité, 6, rue de l'École de médecine, 75006 Paris, France.

julie.gavard@inserm.fr.

précurseurs des cellules endothéliales dérivés du mésoderme et leur association en un réseau vasculaire primitif. Ce processus est principalement observé au cours des étapes précoces du développement embryonnaire, puis lors de la vascularisation des ébauches d'organes. Par la suite, le réseau vasculaire se développe à partir de vaisseaux sanguins préexistants, principalement par prolifération et bourgeonnement des cellules endothéliales, ce qu'on appelle l'angiogenèse.

Le système vasculaire forme un important réseau impliqué dans la distribution d'oxygène et de nutriments aux organes. Les cellules endothéliales qui tapissent la face interne des vaisseaux sont organisées en une fine monocouche et sont étroitement reliées entre elles par des jonctions cellulaires spécialisées. C'est cette barrière endothéliale, en interaction avec les péricytes et une matrice basale différenciée, qui définit les propriétés de la paroi vasculaire (Figure 1A). Elle contrôle finement l'infiltration des protéines plasmatiques et des cellules circulantes dans les tissus sous-jacents [1]. Les échanges entre le compartiment sanguin et les tissus environnants reposent sur l'ouverture et la fermeture des jonctions cellule-cellule, et ils doivent être strictement régulés afin de maintenir l'intégrité vasculaire. L'endothélium mature et quiescent est donc caractérisé par la présence d'un complexe de protéines d'adhérence qui préserve l'homéostasie du compartiment vasculaire [2].

\section{L'angiogenèse tumorale}

Outre leur fonction d'échange, les vaisseaux sanguins contribuent activement aux processus de régénération et de croissance des tissus. Ces fonctions physiologiques peuvent être détournées en conditions pathologiques, notamment dans les cancers. En effet, la croissance de tumeurs solides au-delà de 1 à $2 \mathrm{~mm}^{3}$ est dépendante du développement d'un réseau vasculaire qui leur est consacré. L'angiogenèse 
A

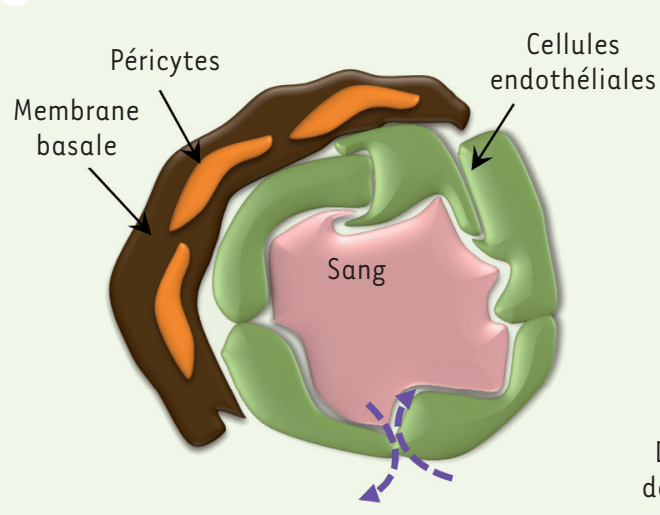

Échanges contrôlés
B

Matrice dégradée
C

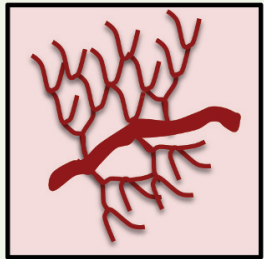

Vaisseaux normaux

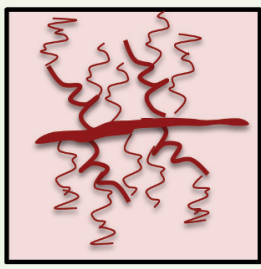

Vaisseaux tumoraux

Figure 1. Comparaison de la vasculature normale et tumorale. A. Les cellules endothéliales tapissent la paroi des vaisseaux et sont étroitement reliées entre elles par des jonctions cellule-cellule. Elles forment, en interaction avec les péricytes et la matrice basale, une barrière qui régule finement le passage des fluides et des macromolécules. B. Dans un contexte tumoral, la perte de l'intégrité des jonctions cellulaires contribue au dysfonctionnement de la barrière endothéliale et à une perméabilité accrue. Les vaisseaux sanguins sont dépourvus de péricytes, dilatés, irréguliers et désorganisés. C. La morphologie globale de l'arbre vasculaire, qui suit une organisation stéréotypée dans les vaisseaux normaux, se caractérise par un profil aberrant des vaisseaux tumoraux.

résulte d'un équilibre entre facteurs activateurs et inhibiteurs. Au cours de la croissance tumorale, un déséquilibre entre signaux proet anti-angiogéniques, appelé switch angiogénique, fait pencher la balance en faveur de la prolifération des cellules vasculaires, de leur migration puis de leur agencement en vaisseaux tumoraux [3]. Ces derniers sont dilatés, irréguliers, perméables et désorganisés (Figure 1C). Ils sont souvent dépourvus de péricytes et de cellules musculaires lisses (Figure IB). La perte de l'intégrité des jonctions cellulaires formées par la VE (vascular endothelial)-cadhérine contribue au dysfonctionnement de la barrière endothéliale et à une perméabilité accrue [2].

\section{Mécanismes cellulaires de l'angiogenèse tumorale}

Les cellules tumorales participent au développement du système vasculaire tumoral, notamment en sécrétant du VEGF (vascular endothelial growth factor), un puissant facteur angiogénique. Outre le mécanisme d'angiogenèse classique qui copie les évènements morphogénétiques du développement embryonnaire, les tumeurs disposent de toute une palette de méthodes originales pour former leurs propres vaisseaux (Figure 2).

\section{Le bourgeonnement ou sprouting}

En conditions tumorales, le bourgeonnement joue un rôle clé dans la croissance des tumeurs primaires et la formation de métastases. Ce processus comporte plusieurs étapes successives qui miment les évènements développementaux de l'angiogenèse. L'activation des cellules endothéliales par divers facteurs de croissance est suivie de la dilatation des vaisseaux pré-existants. Une cellule endothé- liale du front (tip cell) est sélectionnée pour guider la formation du nouveau vaisseau (Figure 2A). En conséquence, la matrice extracellulaire et la membrane basale entourant les cellules endothéliales sont dégradées par des protéases activées localement, comme les MMP (matrix metalloproteinase). Ceci permet aux cellules endothéliales d'envahir la matrice environnante et d'y proliférer. Les cellules migrantes se polarisent et forment un vaisseau sanguin immature, tortueux et perméable (Figure 1C) [4]. Contrairement aux conditions physiologiques, l'angiogenèse tumorale se caractérise par un échec de maturation et d'organisation, résultant en un réseau vasculaire anarchique et une perméabilité élevée.

\section{Angiogenèse par invagination}

Ce processus, observé pour la première fois au cours du remaniement postnatal des capillaires pulmonaires, propose que les vaisseaux préexistants se scindent par formation d'un tissu transvasculaire. Les cellules endothéliales subissent un profond remodelage (Figure 2B): elles augmentent en volume et deviennent très fines. La croissance microvasculaire par invagination est un processus rapide qui peut se produire en quelques heures, voire quelques minutes, indépendamment de la prolifération des cellules endothéliales. L'invagination a souvent lieu après l'angiogenèse classique décrite 


\section{A Bourgeonnement}

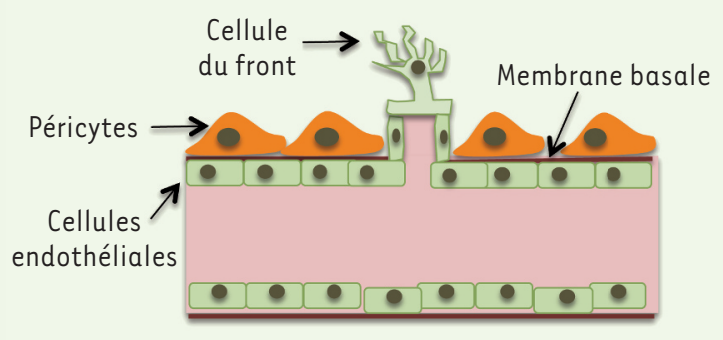

B Mimétisme vasculaire

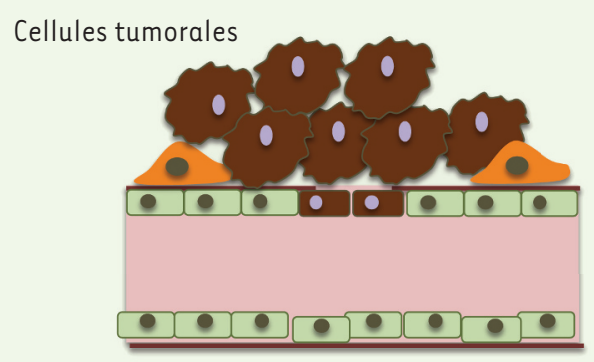

C Invagination

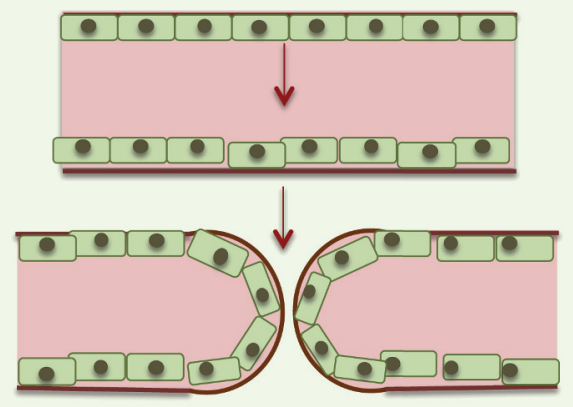

D Transdifférenciation endothéliale

Cellules tumorales à caractère souche

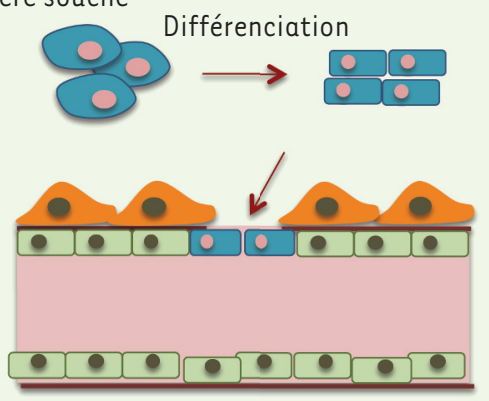

Figure 2. Mécanismes d'angiogenèse tumorale. Les cellules tumorales disposent de toute une palette de méthodes originales pour former leurs propres vaisseaux. Le bourgeonnement (A), qui copie les événements développementaux; le mimétisme vasculaire (B), qui définit la propriété des cellules tumorales à mimer les cellules endothéliales et à s'organiser pour former un réseau vasculaire ; l'invagination (C), qui correspond au mécanisme par lequel les vaisseaux préexistants se divisent en deux pour permettre la formation d'un tissu transvasculaire ; et la transdifférenciation $(D)$, processus par lequel les cellules tumorales à caractère souche acquièrent un phénotype pseudoendothélial et tapissent la paroi interne des vaisseaux.

ci-dessus, et a également été décrite dans les mélanomes et les gliomes $[5,6]$.

\section{Transdifférenciation et mimétisme vasculaire}

Dans certains cancers, les vaisseaux intratumoraux peuvent être constitués en partie par des cellules tumorales qui s'organisent pour former un réseau vasculaire. Les cellules cancéreuses miment ainsi les cellules endothéliales et peuvent former des vaisseaux fonctionnels (Figure 2B). Ce phénomène, appelé mimétisme vasculaire, a été rapporté pour la première fois dans les mélanomes [7]. Une autre possibilité est que les cellules tumorales se dédifférencient et acquièrent un phénotype pseudoendothélial pour former des structures tubulaires de transport (Figure 2D). Ce processus de plasticité cellulaire, appelé transdifférenciation, permet aux cellules tumorales d'élaborer un système de circulation secondaire, indépendamment de l'angiogenèse. Ainsi, les cellules à caractère souche du glioblastome contribuent activement à la vascularisation de la tumeur $[8,9]$. Une étude récente propose à l'inverse que cette population souche, encore mal définie, contienne des cellules à caractère endothélial [10].

\section{Comment la masse tumorale peut-elle moduler la plasticité endothéliale?}

Le microenvironnement, qui comprend divers types cellulaires incluant les cellules endothéliales, les fibroblastes et les cellules inflammatoires (macrophages, mastocytes et neutrophiles), joue un rôle primordial dans la progression et l'invasion tumorales. Nous présentons ici les rôles de ces différents composants dans la modulation de la plasticité de l'endothélium tumoral (Figure 3).

\section{Les cellules tumorales}

Les tumeurs produisent et sécrètent diverses substances angiogéniques dont l'emblématique VEGF, élément clé, non seulement de l'angiogenèse, mais aussi du développement tumoral. Ces fonctions en font une cible de choix dans les thérapies anticancéreuses [4]. Ainsi, l'utilisation du bevacizumab, un anticorps monoclonal dirigé contre le VEGF, conduit à l'épuisement des 


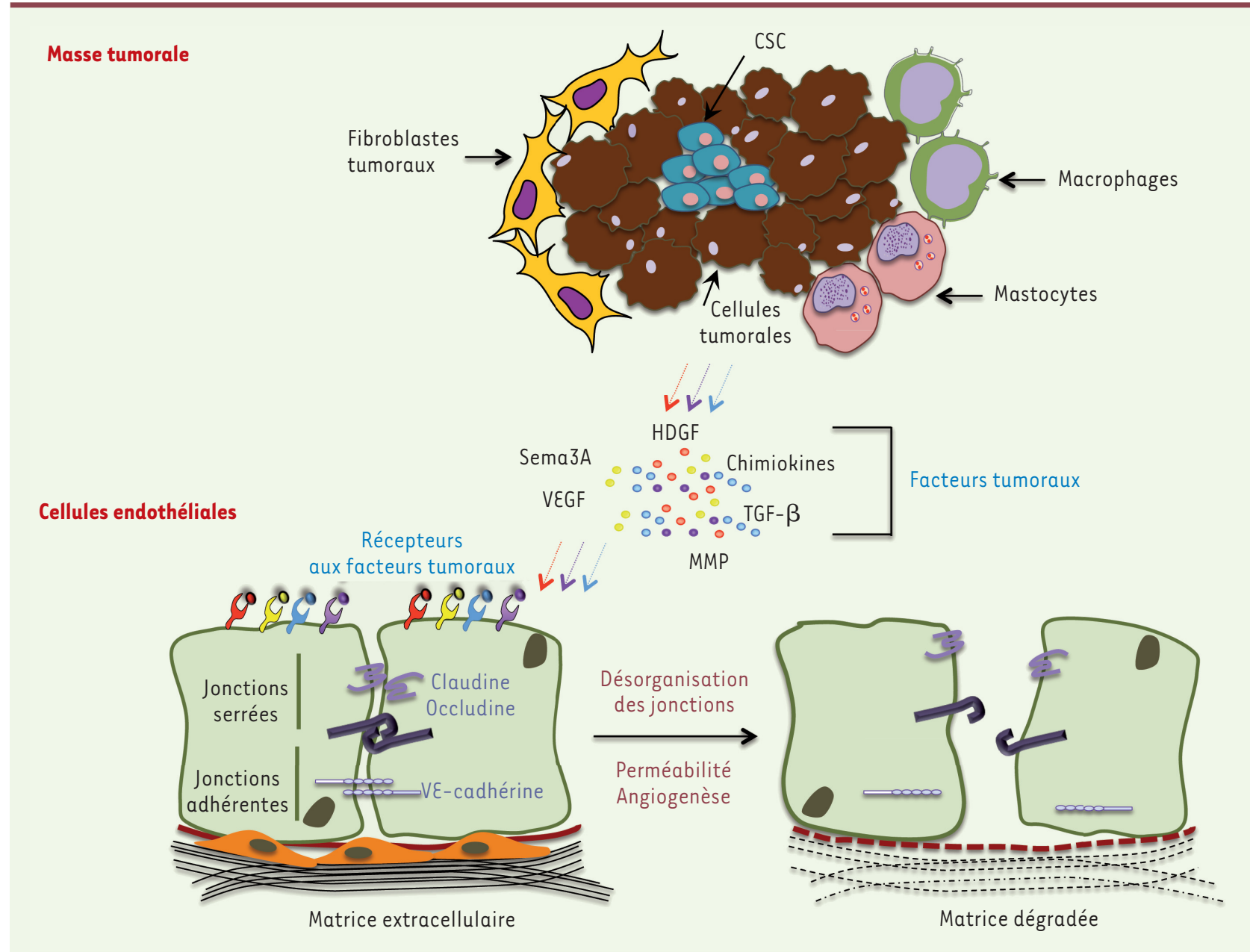

Figure 3. Rôles des facteurs tumoraux dans la modulation de la plasticité endothéliale. Les composants du microenvironnement tumoral (cellules tumorales, fibroblastes et cellules inflammatoires) sécrètent une panoplie de facteurs et chimiokines impliqués dans la modulation de la plasticité de l'endothélium. Ils contribuent à la désorganisation des jonctions cellule-cellule, favorisant ainsi les processus d'angiogenèse et de perméabilité. CSC : cellules souches cancéreuses; HDGF : hepatoma-derived growth factor; Sema3A : Sémaphorine 3A; TGF- $\beta$ : transforming growth factor- $\beta$; MMP : matrix metalloproteinase ; VEGF : vascular endothelial growth factor.

vaisseaux sanguins et l'inhibition de la croissance tumorale dans de nombreux modèles expérimentaux de xénogreffes. Cet anticorps est actuellement utilisé en clinique en traitement de première ligne dans les cancers rénaux et ovariens, et de seconde ligne dans les cancers métastatiques du côlon et du sein, les glioblastomes et les cancers du poumon de type non-small cell lung cancer (source European medical agency) ${ }^{1}$. Le VEGF est également un facteur central de la perméabilité vasculaire [2]. L'exposition des cellules endothéliales au VEGF déclenche une cascade de signalisation qui culmine en l'internalisation de la $V \varepsilon$-cadhérine et le démantèlement des jonctions cellulaires, résultant en une perméabilité accrue [11]. De plus, la surexpression du VEGF est associée à la réduction de l'infiltration des cellules T et à

${ }^{1}$ Voir le numéro thématique «Anticorps monoclonaux en thérapeutique », médecine/sciences $n^{\circ} 12$, vol. 25, décembre 2009. la perte d'adhérence des leucocytes à l'endothélium, ce qui favorise l'échappement de la tumeur à la réponse immunitaire [12].

Outre le VEGF, les cellules tumorales sécrètent du FGF (fibroblast growth factor), du SDF-1 (stroma derived factor- 1 ) et du TGF- $\beta$ (transforming growth factor$\beta$ ). Les effets tumoraux de ce dernier sont nombreux, notamment au cours de la transition épithélio-mésenchymateuse et de l'évasion immune [13]. Le TGF- $\beta$ intervient également dans l'angiogenèse tumorale en induisant l'expression de HIF-l $\alpha$ (hypoxia inducible factor-1 $\alpha$ ) et, donc, du VEGF [14]. Dans les carcinomes mammaires métastatiques, le TGF- $\beta$ augmente l'expression de la protéine angiopoïétine-like 4, qui dissocie les jonctions endothéliales, ce qui permet l'infiltration 
Niche vasculaire

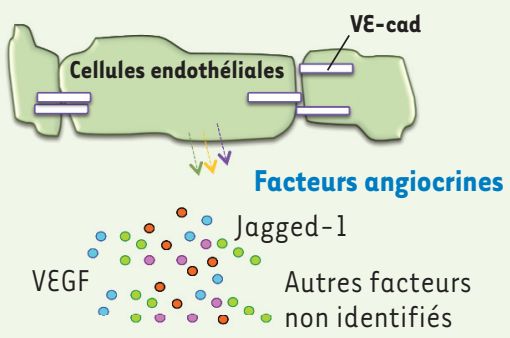

Masse tumorale

A Autorenouvellement des CSC

B Survie et agressivité

C Acquisition du caractère souche

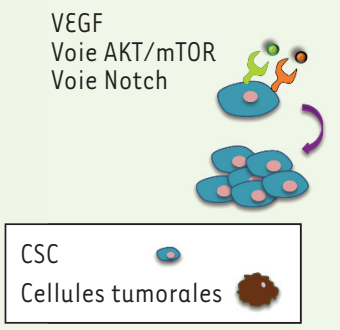

Figure 4. Facteurs angiocrines et tumorigenèse. Les facteurs sécrétés par les cellules endothéliales, ou facteurs angiocrines, sont les acteurs principaux de la progression et de l'invasion tumorales. Ils contribuent, via les voies de signalisation, au renouvellement des cellules tumorales à caractère souche $(A)$, ainsi qu'à l'agressivité de la tumeur (B), et peuvent aussi favoriser l'acquisition d'un phénotype souche des cellules tumorales $(\boldsymbol{C})$.

des cellules tumorales dans les poumons [14]. Les cellules tumorales sécrètent aussi des chimiokines. Par exemple, l'interleukine- 8 (IL-8) contribue dans les gliomes à la désorganisation des jonctions endothéliales et à l'augmentation de la perméabilité [15].

\section{Les cellules tumorales à caractère souche}

Depuis quelques années, l'identification dans plusieurs tumeurs d'une sous-population cellulaire à caractère souche a modifié notre vision du cancer. Ces cellules tumorales à caractère souche (CSC) ont la capacité de s'autorenouveler et de reconstituer la tumeur d'origine dans des modèles de xénogreffes. Les CSC sont réputées plus résistantes aux traitements antitumoraux. Dans les glioblastomes, les CSC se trouvent à proximité des vaisseaux sanguins, suggérant des interactions fonctionnelles entre ces cellules et l'endothélium tumoral [16]. En effet, elles favorisent l'angiogenèse par la sécrétion du VEGF et par leur capacité à se différencier en cellules endothéliales, participant activement à la vascularisation de la tumeur $[8,9,16]$.

Cependant, le VEGF est loin d'être le seul facteur angiogénique sécrété par les CSC. Celles-ci expriment également des cytokines et divers facteurs de croissance dont le HDGF (hepatoma-derived growth factor) impliqué dans la migration des cellules endothéliales cérébrales [17]. Par ailleurs, les CSC sécrètent de la sémaphorine 3A (Sema3A), qui augmente la perméabilité endothéliale en perturbant la stabilité de la VE-cadhérine et l'organisation des jonctions cellule-cellule [18]. Outre cette action sur la perméabilité, la Sema3A apparaît également comme un facteur anti-angiogénique potentiel. $\varepsilon n$ effet, son expression est souvent diminuée au cours de la progression tumorale, en particulier lors du switch angiogénique dans les cancers du pancréas, de l'utérus et les tumeurs cutanées [19]. Ainsi, surexprimer la Sema3A pourrait représenter une nouvelle approche thérapeutique pour freiner le développement des tumeurs, mais il faudrait considérer son effet ambivalent sur la perméabilité vasculaire [18].

\section{Les cellules inflammatoires}

Outre les facteurs proangiogéniques, les cellules tumorales sécrètent des facteurs chimioattractants (GM-CSF [granulocyte-macrophage colony-stimulating factor], IL [interleukine]-3, IL-6), à l'origine du recrutement des macrophages tumoraux TAM (tumor-associated macrophage). Ces derniers jouent un rôle paracrine dans la modulation de l'angiogenèse et la progression tumorale $[13,31](\rightarrow)$.

Au sein du microenvironnement tumoral, les TAM peuvent, soit rem-

$(\rightarrow)$ Voir la Synthèse de S. Garrido-Urbani et al., page 415 de ce numéro plir leurs fonctions immunostimulatrices (type $\mathrm{Ml}$ ), soit exercer des fonctions immunosuppressives (type $M 2$ ), soutenant la progression tumorale par la sécrétion de facteurs proangiogéniques (VEGF, FGF et MMP9). À ce titre, les TAM sont directement impliqués dans la dissémination hématogène des cellules cancéreuses mammaires, ainsi que dans l'invasion métastatique du cancer colorectal [13]. En outre, les polynucléaires neutrophiles et les mastocytes ont été également décrits comme des acteurs de l'angiogenèse via une action paracrine [20]. Enfin, dans le myélome multiple, les monocytes ont la capacité de se différencier en cellules endothéliales, favorisant ainsi l'angiogenèse tumorale par transdifférenciation [21].

\section{Le stroma tumoral et la matrice extracellulaire}

Les cellules stromales du microenvironnement tumoral exercent une fonction importante lors du switch angiogénique. En effet, les fibroblastes tumoraux sont des composants cellulaires essentiels de la niche tumorale. Ils sécrètent des cytokines, des protéases et des 
chimiokines, favorisant l'angiogenèse $[14,22,32$, 33] $(\rightarrow)$.

La matrice extracellulaire (ECM), dont la composition et l'assemblage sont complexes, peut être modifiée par les cellules tumorales, transformant ainsi leur microenvironnement à leur avantage $[23,34](\rightarrow)$.

Outre qu'elle fournit des facteurs de croissance, la $(\rightarrow)$ Voir les Synthèses de J. Albrengues et al., et de V. Provot, pages 366 et 391 de ce numéro

$\rightarrow$ Voir la Synthèse de $\varepsilon$. Buache et M.C. Rio, page 385 de ce numéro niche favorise la progression tumorale par réarrangement de la MEC. Les MMP, dont la production est augmentée dans la majorité des cancers, clivent les molécules d'adhérence cellulaire et dégradent les protéines de la matrice, aboutissant à une prolifération cellulaire et une angiogenèse accrues, ainsi qu'à une augmentation du pouvoir métastasique. Les interactions entre cellules cancéreuses et endothélium tumoral regroupent des processus qui demeurent encore complexes. En effet, les cellules endothéliales peuvent aussi moduler le phénotype et les propriétés des cellules cancéreuses.

\section{Quel est le rôle des facteurs endothéliaux ou angiocrines dans la modulation des caractéristiques d'une tumeur?}

Les cellules souches normales résident dans une niche soutenue par une unité vasculaire ; l'endothélium fournit une concentration favorable en oxygène, facteurs de croissance et protéines d'adhérence, et crée un microenvironnement de protection. Dans un modèle non néoplasique, celui de la niche hématopoïétique, les cellules souches hématopoïétiques (CSH) résident à proximité des cellules endothéliales et ces dernières contribuent à la régulation de la division asymétrique des CSH $[24,35](\rightarrow)$. De la même manière, les cellules endothéliales sinusoïdales hépatiques $\rightarrow$ Voir la Synthèse de J.C. Chomel et al., page 452 de ce numéro sécrètent des facteurs angiocrines capables d'induire et de favoriser la régénération du foie en réponse à une hépatectomie partielle [25].

À l'instar des cellules souches normales, les cellules souches cancéreuses résident dans une niche vasculaire, et l'endothélium tumoral pourrait aussi jouer un rôle crucial dans l'initiation et la progression tumorales (Figure 4). Ainsi, les cellules endothéliales semblent être impliquées dans le maintien de la population de CSC [16, 26]. Dans des tumeurs vascularisées, telles que les glioblastomes, il existe une relation étroite entre les CSC et les vaisseaux sanguins, et les cellules endothéliales sont capables de maintenir des cellules dérivées de tumeur dans un état de type souche, promouvant ainsi leur potentiel tumorigène. Ainsi, l'inhibition de l'angiogenèse et l'épuisement des vaisseaux sanguins par le bevacizumab réduisent la proportion de CSC et inhibent la croissance tumorale [16]. En outre, l'inhibition du récepteur VEGFR2 ou de son co-récepteur, la neuropiline-1, dans un modèle murin de tumeurs cutanées altère non seulement l'angiogenèse tumorale, mais aussi la division et l'autorenouvellement des CSC, contribuant ainsi à la régression tumorale [22].

Les cellules endothéliales seraient également impliquées dans l'autorenouvellement des CSC via la signalisation Notch et l'inhibition de la différenciation des cellules souches. $\varepsilon$ n effet, les cellules endothéliales produisent l'oxyde nitrique (NO) qui active la voie Notch et contribue au maintien du phénotype souche des cultures primaires de gliomes. De plus, la perte d'expression de l'enzyme eNOS, qui produit le NO périvasculaire, ralentit la formation tumorale via une inhibition de la voie Notch [27]. Par ailleurs, notre laboratoire a montré que les cellules endothéliales cérébrales sont impliquées dans le maintien des propriétés souches des CSC via la voie AKT (protéine kinase B)/mTOR (mammalian target of rapamycin) [26]. Des études protéomiques plus détaillées suggèrent que les cellules endothéliales sécrètent des facteurs spécifiques qui pourraient agir de façon paracrine pour maintenir la population de CSC (Galan-Moya et Gavard, résultats non publiés).

Les cellules endothéliales seraient également impliquées dans l'activation constitutive de la voie $N F-\kappa B$ et la surexpression de protéines antiapoptotiques dans un modèle de leucémie lymphoïde chronique, contribuant ainsi à la survie et à l'agressivité de la tumeur [28]. Enfin, dans les cancers colorectaux, les cellules endothéliales sécrètent, sous l'action de la métalloprotéinase ADAM17 (A disintegrin and metalloproteinase domain 17), une forme soluble de la protéine jagged-1 (un ligand de Notch). Libérée par les cellules endothéliales, la forme clivée de jagged-1 active la voie Notch dans les cellules souches cancéreuses par une voie paracrine. Cette voie de signalisation inédite favorise le phénotype souche des cellules tumorales, la résistance aux chimiothérapies, la progression tumorale et la formation de métastases [29]. L'ensemble de ces données récentes suggèrent un rôle essentiel des facteurs angiocrines et des échanges entre l'endothélium et les cellules de la masse tumorale dans l'initiation et la progression tumorales.

\section{Conclusion et perspectives}

Les interactions bidirectionnelles entre les cellules endothéliales et la masse tumorale commencent à être explorées en détail dans des modèles cellulaires et animaux. II s'agit d'un véritable dialogue qui bénéficie à l'ensemble des composants de la tumeur. Ainsi, il semblerait judicieux de s'orienter vers des thérapies combinées antitumorales et anti-angiogéniques. À ce titre, l'utilisation du bevacizumab couplé à la chimiothérapie permet une prolongation de la survie des patients [30], bien que les bénéfices sont encore sujets à questionnements [36]. De nouvelles cibles thérapeutiques capables d'interrompre ce dialogue ou de le détourner doivent maintenant être développées. $\diamond$

\section{SUMMARY}

Blood vessels in cancer: can't stop whispering

Tumor initiation and progression is a complex process in which cells acquire aberrant proliferation, survival and 
migration properties, and the ability to form a dedicated blood vessel network. Recent studies highlighted the existence of an active crosstalk between endothelial cells and the tumor mass. Indeed, cancer stem-like cells have been identified and found in the vicinity of blood vessels, and the latter has been proposed to act as a feeding bed for tumors, especially governing the fate of cancer stem-like cells. Here, we present an overview of the direct interplay between endothelial and cancer cells. We will first introduce the mechanisms involved in tumor angiogenesis. How the microenvironment impacts on endothelial plasticity will next be detailed, focusing on the tumor main secreted factors. Finally, the role of the vascular niche and its angiocrine factors in tumorigenesis will be addressed. $\diamond$

\section{LIENS D'INTÉRÊT}

Les auteurs déclarent n'avoir aucun lien d'intérêt concernant les données publiées dans cet article.

\section{REMERCIEMENTS}

Les auteurs remercient les membres du laboratoire de Julie Gavard à l'Institut Cochin. Nous tenons à remercier la Fondation ARC pour la recherche sur le cancer, la Ligue nationale contre le cancer, et la Fondation pour la recherche médicale pour leurs soutiens financiers.

\section{RÉFÉRENCES}

1. Vestweber D, Winderlich M, Cagna G, Nottebaum AF. Cell adhesion dynamics at endothelial junctions: VE-cadherin as a major player. Trends Cell Biol $2009 ; 19: 8-15$.

2. Gavard J, Gutkind JS. La VE-cadhérine prend des chemins de traverse. Med Sci (Paris) 2007 ; 23 : 119-21.

3. Bensimon J. Le switch angiogénique ou comment réveiller les cellules tumorales dormantes. Med Sci (Paris) $2012 ; 28: 1069-71$.

4. Carmeliet P, Jain RK. Molecular mechanisms and clinical applications of angiogenesis. Nature $2011 ; 473: 298-307$.

5. Ribatti D, Nico B, Floris C, et al. Microvascular density, vascular endothelial growth factor immunoreactivity in tumor cells, vessel diameter and intussusceptive microvascular growth in primary melanoma. Oncol Rep $2005 ; 14: 81-4$.

6. Nico B, Crivellato $\varepsilon$, Guidolin D, et al. Intussusceptive microvascular growth in human glioma. Clin Exp Med $2010 ; 10: 93-8$.

7. Maniotis AJ, Folberg R, Hess A, et al. Vascular channel formation by human melanoma cells in vivo and in vitro: vasculogenic mimicry. Am J Pathol $1999 ; 155$ : 739-52.

8. Ricci-Vitiani L, Pallini R, Biffoni M, et al. Tumour vascularization via endothelial differentiation of glioblastoma stem-like cells. Nature $2010 ; 468: 824-8$.

9. Wang R, Chadalavada K, Wilshire J, et al. Glioblastoma stem-like cells give rise to tumour endothelium. Nature $2010 ; 468: 829-33$.

10. Golebiewska A, Bougnaud S, Stieber D, et al. Side population in human glioblastoma is nontumorigenic and characterizes brain endothelial cells. Brain $2013 ; 136: 1462-75$.

11. Gavard J, Gutkind JS. VEGF controls endothelial-cell permeability by promoting the beta-arrestindependent endocytosis of VE-cadherin. Nat Cell Biol 2006; 8 : 1223-34.

12. Dirkx AE, Oude Egbrink MG, Kuijpers MJ, et al. Tumor angiogenesis modulates leukocyte-vessel wall interactions in vivo by reducing endothelial adhesion molecule expression. Cancer Res $2003 ; 63: 2322-9$.

13. Pollard JW. Tumour-educated macrophages promote tumour progression and metastasis. Nat Rev Cancer $2004 ; 4: 71-8$.

14. Zhang $\mathrm{H}$, Wong $\mathrm{CC}$, Wei $\mathrm{H}$, et al. HIF-1-dependent expression of angiopoietin-like 4 and LICAM mediates vascular metastasis of hypoxic breast cancer cells to the lungs. Oncogene $2012 ; 31: 1757-70$.
15. Dwyer J, Hebda JK, Le Guelte A, et al. Glioblastoma cell-secreted interleukin-8 induces brain endothelial cell permeability via CXCR2. PLoS One $2012 ; 7$ : e45562.

16. Calabrese $C$, Poppleton $H$, Kocak M, et al. A perivascular niche for brain tumor stem cells. Cancer Cell $2007 ; 11: 69-82$.

17. Thirant C, Galan-Moya EM, Dubois LG, et al. Differential proteomic analysis of human glioblastoma and neural stem cells reveals HDGF as a novel angiogenic secreted factor. Stem Cells $2012 ; 30: 845-53$.

18. Le Guelte A, Galan-Moya EM, Dwyer J, et al. Semaphorin $3 \mathrm{~A}$ elevates endothelial cell permeability through PP2A inactivation.J Cell Sci 2012 ; 125 : 4137-46.

19. Maione F, Molla F, Meda C, et al. Semaphorin $3 \mathrm{~A}$ is an endogenous angiogenesis inhibitor that blocks tumor growth and normalizes tumor vasculature in transgenic mouse models. J Clin Invest $2009 ; 119: 3356-72$.

20. Soucek $L$, Lawlor $\varepsilon R$, Soto $D$, et al. Mast cells are required for angiogenesis and macroscopic expansion of Myc-induced pancreatic islet tumors. Nat Med $2007 ; 13: 1211-8$

21. Chen $\mathrm{H}$, Campbell RA, Chang $\mathrm{Y}$, et al. Pleiotrophin produced by multiple myeloma induces transdifferentiation of monocytes into vascular endothelial cells: a novel mechanism of tumor-induced vasculogenesis. Blood 2009; 113 : 1992-2002.

22. Beck B, Driessens G, Goossens S, et al. A vascular niche and a VEGF-Nrpl loop regulate the initiation and stemness of skin tumours. Nature 2011 ; 478 : 399-403.

23. Koninger J, Giese T, di Mola FF, et al. Pancreatic tumor cells influence the composition of the extracellular matrix. Biochem Biophys Res Commun $2004 ; 322: 943-9$.

24. Kiel MJ, Yilmaz $\mathrm{OH}$, Iwashita T, et al. SLAM family receptors distinguish hematopoietic stem and progenitor cells and reveal endothelial niches for stem cells. Cell $2005 ; 121: 1109-21$.

25. Ding BS, Nolan DJ, Butler JM, et al. Inductive angiocrine signals from sinusoidal endothelium are required for liver regeneration. Nature $2010 ; 468: 310-5$.

26. Galan-Moya EM, Le Guelte A, Lima Fernandes $\varepsilon$, et al. Secreted factors from brain endothelial cells maintain glioblastoma stem-like cell expansion through the mTOR pathway. EMBO Rep 2011; $12: 470-6$

27. Charles $N$, Ozawa T, Squatrito $M$, et al. Perivascular nitric oxide activates notch signaling and promotes stem-like character in PDGF-induced glioma cells. Cell Stem Cell $2010 ; 6: 141-52$

28. Buggins $A G$, Pepper $C$, Patten PE, et al. Interaction with vascular endothelium enhances survival in primary chronic lymphocytic leukemia cells via NF-kappaB activation and de novo gene transcription. Cancer Res $2010 ; 70: 7523-33$.

29. Lu J, Ye X, Fan F, et al. Endothelial cells promote the colorectal cancer stem cell phenotype through a soluble form of Jagged-1. Cancer Cell $2013 ; 23: 171-85$.

30. Norden AD, Young GS, Setayesh $K$, et al. Bevacizumab for recurrent malignant gliomas: efficacy, toxicity, and patterns of recurrence. Neurology $2008 ; 70: 779-87$

31. Garrido-Urbani S, Jaquet V, Imhof BA. ERO, NADPH oxydases et vascularisation des tumeurs. Med Sci (Paris) $2014 ; 30: 415-21$.

32. Albrengues J, Meneguzzi G, Gaggioli C. L'invasion des cellules tumorales : quand les fibroblastes s'en mêlent. Med Sci (Paris) 2014 ; $30: 391-7$.

33. Provot $\mathrm{S}$. Contrôle de la croissance et de la dissémination tumorales par le microenvironnement : certitudes et hypothèses émergentes. Med Sci (Paris) $2014 ; 30: 366-71$

34. Buache $\varepsilon$, Rio MC. Le stroma tumoral, un terreau fertile pour la cellule cancéreuse. Med Sci (Paris) 2014 ; $30: 385-90$.

35. Chomel JC, Aggoune D, Sorel N, Turhan AG. Leucémie myéloïde chronique un modèle de dialogue entre la cellule souche leucémique et la niche hématopoïétique. Med Sci (Paris) 2014 ; 30 : 452-61.

36. Chinot OL, Wick W, Mason W, et al. Bevacizumab plus radiotherapy-temozolomide for newly diagnosed glioblastoma. N Engl J Med 2014 ; 20 : 709-22.

\section{TIRÉS À PART}

J. Gavard

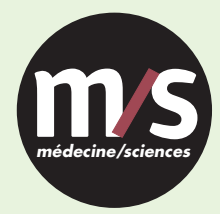

Tarifs d'abonnement $\mathrm{m} / \mathrm{s}-2014$

Abonnez-vous

à médecine/sciences
$>$ Grâce à $m / s$, vivez en direct les progrès des sciences biologiques et médicales

Bulletin d'abonnement page 470 dans ce numéro de $\mathrm{m} / \mathrm{s}$

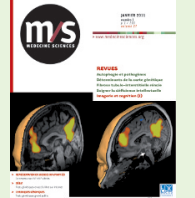

